\title{
Early and Long-Term Mortality of the Clinical Subtypes of Myocarditis
}

\author{
Makoto Kodama, MD; Hirotaka Oda, MD*; Masaaki Okabe, MD**; \\ Yoshifusa Aizawa, MD; Tohru Izumi, MD ${ }^{\dagger}$
}

\begin{abstract}
The frequency of myocarditis and the prognosis for patients remains uncertain and, moreover, the clinical classification of myocarditis is controversial. From 1985 to 2000, 71 adult patients with clinically suspected myocarditis were admitted to 11 cardiovascular centers. Of these, 48 cases had histology proven myocarditis: 41 cases of lymphocytic myocarditis, 6 of giant cell myocarditis and 1 of eosinophilic myocarditis. Myocarditis was classified as acute ( 30 cases) or chronic ( 18 cases) according to the onset of the disease, and acute myocarditis was further categorized into common or fulminant type depending on whether or not patients required mechanical circulatory support in the management of heart failure ( 9 and 21 cases, respectively). Chronic myocarditis was divided into 3 subgroups: a persistent type lasting over 3 months after distinct onset ( 3 cases), a recurrent type (2 cases) and a latent form (13 cases). The early mortality of these 5 subtypes of myocarditis were acute common $22 \%$, acute fluminant $43 \%$, chronic persistent $33 \%$, chronic recurrent $50 \%$, and chronic latent $38 \%$. The overall early mortality of all patients with myocarditis was $38 \%$ in spite of aggressive treatment during hospitalization. On the other hand, the long-term prognosis of patients with myocarditis was favorable; only 4 cases, who survived the active phase, died in the late phase: 1 had fulminant myocarditis and the other 3 had the chronic latent form. Thus, the early mortality of patients with myocarditis was very high regardless of the subtype, but if patients can survive the active phase, they have a favorable prognosis except with the chronic latent form. (Jpn Circ J 2001; 65: 961-964)
\end{abstract}

Key Words: Chronic myocarditis; Clinical subtype; Fulminant myocarditis; Latent myocarditis; Myocarditis

M yocarditis is an inflammatory heart muscle disease, the major causes of which are viral infection and allergic/autoimmune reaction! The clinical manifestation varies from flu-like symptoms to cardiogenic shock and the classification of myocarditis has not been universally standardized?,3 Acute myocarditis is usually defined having a distinct onset and the term 'fulminant myocarditis' is used for acute myocarditis with cardiogenic shock requiring mechanical circulatory support systems.-6 On the other hand, there are other classifications based on endomyocardial biopsy findings ${ }^{-9}{ }^{-9}$ Fulminant myocarditis has been recently reported to have a more favorable prognosis than acute myocarditis in the USA ${ }^{10}$ so the recognition and classification of myocarditis may vary among races and countries. The incidence and prognosis of the clinical subtypes of myocarditis were investigated in this study.

\section{Methods}

\section{Patients}

From 1985 to 2000, 71 patients were admitted to 11 hospitals and diagnosed as having clinical myocarditis

(Received April 23, 2001; revised manuscript received July 23, 2001; accepted July 27, 2001)

Division of Cardiology, Niigata Graduate School of Medicine \& Dental Science, Niigata, *Niigata City General Hospital, Niigata, **Tachikawa General Hospital, Nagaoka and Department of Medicine, Kitasato University School of Medicine, Sagamihara, Japan Mailing address: Makoto Kodama, MD, Division of Cardiology, Niigata Graduate School of Medicine \& Dental Science, 1-754 Asahimachi, Niigata 951-8510, Japan. E-mail: kodamama@med. niigata-u.ac.jp based on the following findings. There were cardiac symptoms, such as congestive heart failure or life-threatening arrhythmias, together with inflammatory signs or accumulation of ${ }^{67} \mathrm{Ga}$-citrate in the heart. There were serial changes in the serum concentration of creatine kinase and on the ECG in accord with the clinical course. Patients showed varying degrees of left ventricular dysfunction, but coronary artery disease was ruled out in the majority of patients by coronary angiography.

Of these 71 cases, 48 had histology proven myocarditis (30 male, 18 female). The age of the patients ranged from 16 to 71 years (mean, 50.1 \pm 16.7 ). The histologic diagnosis of myocarditis was principally based on the Dallas criteria $^{11}$ and was confirmed by endomyocardial biopsy in 37 patients and by autopsy in 11 cases. There were 41 cases of lymphocytic myocarditis, 6 of giant cell myocarditis and 1 of eosinophilic myocarditis. Giant cell myocarditis was distinguished from cardiac sarcoidosis by the absence of extra-cardiac involvement and the acute inflammatory findings on histology.

\section{Clinical Subtypes of Myocarditis}

Myocarditis was classified into acute (30 cases) or chronic type (18 cases) according to the onset of the disease ${ }^{12}$ Acute myocarditis was further categorized into common or fulminant type depending on whether or not patients required mechanical circulatory support in the management of heart failure ${ }^{13}$ : 9 cases were classified as acute common type and the other 21 cases were diagnosed as fulminant. Chronic myocarditis was divided into 3 subtypes: a persistent type that continued more than 3 months after distinct onset ( 3 cases), a recurrent type (2 
Table 1 Characteristics of the Clinical Subtypes of Myocarditis

\begin{tabular}{|c|c|c|c|c|c|c|c|c|c|}
\hline \multirow{2}{*}{ Subtype } & \multirow{2}{*}{ Onset } & \multirow{2}{*}{$n$} & \multirow{2}{*}{ Male/female } & \multirow{2}{*}{ Age (years) } & \multicolumn{2}{|c|}{ Diagnosis confirmed by } & \multicolumn{3}{|c|}{ Histologic classification } \\
\hline & & & & & Biopsy & Autopsy & Lymphocytic & Giant cell & Eosinophilic \\
\hline \multicolumn{10}{|l|}{ Acute } \\
\hline Common & Distinct & 9 & $5 / 4$ & $44.7 \pm 19.2$ & 7 & 2 & 8 & 1 & 0 \\
\hline Fulminant & Distinct & 21 & $15 / 6$ & $51.8 \pm 17.0$ & 17 & 4 & 20 & 1 & 0 \\
\hline \multicolumn{10}{|l|}{ Chronic } \\
\hline Persistent & Distinct & 3 & $2 / 1$ & $51.3 \pm 13.2$ & 2 & 1 & 3 & 0 & 0 \\
\hline Recurrent & Indistinct & 2 & $1 / 1$ & $57.0 \pm 18.4$ & 2 & 0 & 1 & 1 & 0 \\
\hline Latent & Indistinct & 13 & $7 / 6$ & $49.6 \pm 16.3$ & 9 & 4 & 9 & 3 & 1 \\
\hline
\end{tabular}
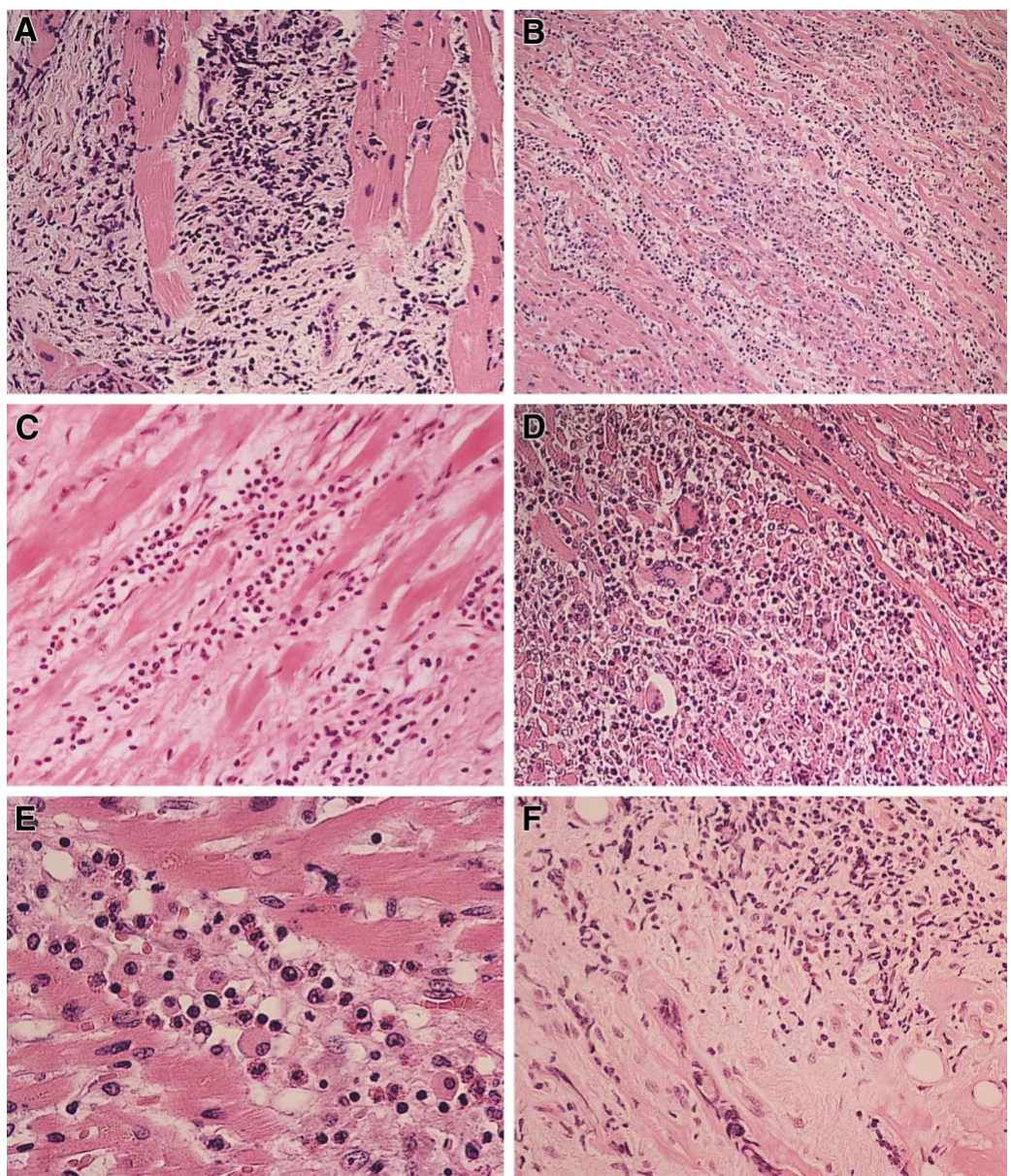

Fig 1. (A) Endomyocardial biopsy from a 49year-old man who developed chest pain and complete atrioventricular block. He was diagnosed as acute common, lymphocytic myocarditis $(\times 200)$. (B) Autopsy finding from a 24-year-old woman with high fever and cardiogenic shock. Her diagnosis was acute fulminant, lymphocytic myocarditis $(\times 100)$. (C) Autopsy finding of a 44year-old woman who had been diagnosed as chronic latent, giant cell myocarditis from the initial endomyocardial biopsy. Five months after the initial episode, congestive heart failure caused by progressive left ventricular dysfunction developed and the final diagnosis was chronic recurrent, giant cell myocarditis $(\times 200)$. (D) Autopsy finding of a 19-year-old woman who died suddenly. She was diagnosed as chronic latent, giant cell myocarditis $(\times 200)$. (E) Autopsy finding from 54-yearold woman. There was significant infiltration of eosinophils and the diagnosis was chronic latent, eosinophilic myocarditis $(\times 400)$. (F) Endomyocardial biopsy finding of a 38-year-old man with left ventricular dysfunction and frequent ventricular premature beats. He was diagnosed as chronic latent, lymphocytic myocarditis $(\times 200)$. cases) and a latent form without distinct onset (13 cases). Patients with the recurrent and latent forms presented with congestive heart failure or life threatening arrhythmias with left ventricular dysfunction, and endomyocardial biopsies revealed inflammatory mononuclear cell infiltration and myocardial degeneration. In 2 cases of the recurrent type of myocarditis, the congestive heart failure were successfully managed by conventional therapy alone or with steroid therapy, after which the patients were discharged from hospital even though the left ventricular dysfunction persisted. However, both cases had a recurrence of myocarditis with congestive heart failure during the follow-up period. Our study population was biased toward severe cases of myocarditis, because less severe patients would not be admitted to hospital and would not undergo cardiac catheterization and endomyocardial biopsy. The majority of cases of the acute common type may have been lost from this study.

Statistical Analysis

The frequency of the mortality rate among the 5 groups was compared using chi-square analysis.

\section{Results}

Patient characteristics of each clinical subtype are shown in Table 1. There was a high prevalence of both the acute fulminant and chronic latent forms in this study, whereas the chronic persistent and chronic recurrent forms were scarce. Males were predominant in every subtype. The ages of the patients in each subtype were not different. From the histologic classification, lymphocytic myocarditis was predominant in every group. The chronic latent form included a significant number of patients with giant cell 
Table 2 Mortality of the Clinical Subtypes of Myocarditis

\begin{tabular}{lccccc}
\hline \hline Subtype & $n$ & Early death & Early mortality & Late death & Overall mortality \\
\hline Acute & & & & & \\
$\quad$ Common & 9 & 2 & $22 \%$ & 0 & $22 \%$ \\
$\quad$ Fulminant & 21 & 9 & $43 \%$ & 1 & $48 \%$ \\
Chronic & 3 & 1 & $33 \%$ & 0 & $33 \%$ \\
$\quad \begin{array}{l}\text { Persistent } \\
\text { Recurrent }\end{array}$ & 2 & 1 & $50 \%$ & 0 & $50 \%$ \\
Latent & 13 & 5 & $38 \%$ & 3 & $62 \%$ \\
\hline
\end{tabular}

myocarditis and eosinophilic myocarditis, although giant cell myocarditis was not restricted to specific clinical subtypes. The histologic findings of representative cases of the various clinical forms of myocarditis are shown in Fig 1.

The time from symptomatic onset to admission to a cardiovascular center could be determined in both the acute common and acute fulminant subtypes (6.4 and 6.5 days, respectively). In the acute common group, the concentration of creatine kinase on admission and the peak value during the clinical course were $631 \pm 534$ and $759 \pm 384$ IU/L, respectively, and those of the acute fulminant type were $1,666 \pm 1,787$ and 4,868 7 7,484 IU/L, respectively. All patients with the fulminant type had intra-aortic balloon pumping (IABP) and 12 patients required percutaneous cardiopulmonary support (PCPS). One case of chronic persistent myocarditis and 3 cases of the chronic latent type were also managed with IABP, and PCPS was used in 2 patients with chronic latent myocarditis.

The number of deaths during the clinical course and the mortality rate of each subtype are shown in Table 2. There was no statistical difference in early and overall mortality among the 5 groups. The early mortality of every group was high, from $22 \%$ to $50 \%$, but late death occurred in only the acute fulminant and the chronic latent subtypes. In particular, 3 of 8 cases in the chronic latent group, who survived and were discharged after initial hospitalization, died during follow up. Thus, the early mortality of patients with myocarditis was high regardless of clinical subtypes and there was a high prevalence of late deaths in patients with chronic latent myocarditis.

\section{Discussion}

The mortality rates of the clinical subtypes of myocarditis were investigated in Japanese patients. When patients had severe congestive heart failure or life-threatening arrythmias, the early mortality was high regardless of the clinical subtype and despite aggressive treatment. However, if patients survived the active phase, their prognosis was generally favorable, except if they had the chronic latent form.

There are 3 classification systems for myocarditis. The first is an etiology based classification, such as viral infection, toxic exposure, allergic/autoimmune and idiopathic. The second is a histology based classification, such as lymphocytic, eosinophilic and giant cell myocarditis. The third classification is based on the clinical course, as described in the present study. The links between the 3 classifications are as yet obscure. An etiology based classification is the most valuable in clinical practice, but the majority of patients will be classified as idiopathic because the etiology of every patient cannot be defined. A histology based classification is also valuable in clinical practice and there may be a loose linkage with the etiology-based classification. However, there is also a significant limitation in this classification. Endomyocardial biopsy is not easy and not convenient, especially for patients with mild symptoms. In addition, the sensitivity of endomyocardial biopsy for myocarditis is not satisfactorily high and furthermore, because of sampling error some patients with giant cell myocarditis may be judged as lymphocytic myocarditis. On the other hand, a classification based on the clinical course can be used in all patients with myocarditis, but until now, there has not been a report concerning the linkage between clinical subtype and histologic classification. The results of our study suggest that there is not a definite linkage between clinical subtype and histologic classification, but that loose linkages may exist between acute myocarditis and the lymphocytic form, and between the chronic latent form and giant cell myocarditis. Furthermore, this study revealed the prognosis of the clinical subtypes.

A recent report suggested that in the USA fulminant myocarditis had a more favorable prognosis than acute myocarditis 10 However, the classification of myocarditis in the USA differs from the Japanese classification. Fulminant myocarditis is defined as myocarditis with distinct onset, which would correspond to the acute common and the acute fulminant forms of the Japanese classification, and acute myocarditis is defined as the disease with indistinct onset, which would correspond to the chronic latent form in our study. The prognosis of patients with myocarditis in Japan was similar to the mortality in the USA except for differences in nomenclature.

The therrapeutic strategies were not uniform in this patient population because there is not a specific and standardized therapy for myocarditis 14,15 Steroid therapy is not indicated for patients with acute common and acute fulminant myocarditis ${ }^{16}$ but a few patients with acute fulminant myocarditis did receive steroid therapy at the clinical stage of multiple organ failure. A significant number of patients with chronic myocarditis were treated by steroid hormones, but we could not estimate the efficacy of this therapy for chronic myocarditis because we have no comparable control. If the clinical classification of myocarditis is widely accepted, the efficacy of steroid therapy or other immunosuppressive therapy for each subtype of chronic myocarditis will be able to be clarified.

Patients with the chronic latent form had a high mortality during the follow-up period, which may be the result of the absence of specific therapy and the difficulty in estimating disease activity in the chronic latent form. Therefore, patients with chronic latent myocarditis should be managed cautiously even when their initial condition has stabilized.

\section{Study Limitations}

Our patient population may have been biased to severe 
cases because in Japan endomyocardial biopsy is not routinely performed and so the majority of less severe patients with acute common myocarditis would not undergo cardiac catheterization for endomyocardial biopsy. Furthermore, this study was retrospective and contained autopsy-proven myocarditis, which may have worsened the calculated mortality of myocarditis.

\section{Conclusion}

The clinical manifestation of myocarditis varies from mild, flu-like symptoms to severe, life-threatening heart failure. Patients with severe symptoms should be managed cautiously during the active phase because of significant mortality. The clinical classification by the Japanese Circulation Society (JCS) Task Force Committee is convenient to use and will be valuable for assessing subtypespecific therapeutic strategies.

\section{Acknowledgments}

We gratefully acknowledge the following doctors for their cooperation in this study.

Niigata City General Hospital, Norio Higuma, MD, Tsutomu Miida, MD, Kazuyoshi Takahashi, MD; Niigata Kobari Hospital, Hideaki Otsuka, MD, Mitsuru Oshima, MD, Yasushi Miyakita, MD; Kido Hospital, Takashi Tsuda, MD, Toshio Yamaguchi, MD, Takefumi Miyajima, MD; Tsubame Rosai Hospital, Seiichi Miyajima, MD; Shinrakuen Hospital, Akihiro Yokoyama, MD, Makiko Tsutsui, MD; Niigata Prefectural Shibata Hospital, Kaoru Suzuki, MD, Yasuhiko Tanabe, MD, Eiichi Ito, MD, Tachikawa General Hospital, Masahito Sato, MD, Junji Ishiguro, MD, Minoru Takahashi, MD, Hitoshi Kitazawa, MD; Nagaoka Red Cross Hospital, Tsuneo Nagai, MD, Katsuya Ebe, MD, Toshio Fujita, MD; Nagaoka Center Hospital, Makihiko Saeki, MD; Niigata Prefectural Center Hospital, Fumiaki Masani, MD, Osamu Ogawa, MD; Department of Clinical Pharmacology, Niigata College of Pharmacy, Kenichi Watanabe, MD, and First Department of Internal Medicine, Niigata University Hospital, Haruo Hanawa, MD, Kiminori Kato, MD, Yuji Okura, MD, Satoru Hirono, MD, Takaaki Shiono, MD, Koichi Fuse, MD, Masahiro Ito, MD, Hitoshi Tachikawa, MD, Tsuyoshi Yoshida, MD, Manabu Hayashi, MD, Satoru Abe, MD, Hui Liu, MD, and Raafat Elnaggar, $M D$.

\section{References}

1. Kawai C: From myocarditis to crdiomyopathy: Mechanisms of inflammation and cell death: Learning from the past for the future.
Circulation 1999; 99: 1091-1100

2. Fenoglio JJ, Ursell PC, Kellogg CF, Drusin RE, Weiss MB: Diagnosis and classification of myocarditis by endomyocardial biopsy. $N \mathrm{Engl}$ J Med 1983; 308: 12-18

3. Dec GW, Palacios IF, Fallon JT, Aretz HT, Mills J, Lee DCS, et al: Active myocarditis in the spectrum of acute dilated cardiomyopathies: Clinical features, histologic correlates, and clinical outcome. N Engl J Med 1985; 312: 885-890

4. Morishima I, Sassa H, Sone T, Tsuboi H, Kondo J, Koyama T: A case of fulminant myocarditis rescued by long-term percutaneous cardiopulmonary support. Jpn Circ J 1994; 58: 433-438

5. Kawahito K, Murata S, Yasu T, Adachi H, Ino T, Saito M, et al: Usefulness of extracorporeal membrane oxygenation for treatment of fulminant myocarditis and circulatory collapse. Am J Cardiol 1998; 82: $910-911$

6. Kato S, Morimoto S, Hiramitsu S, Nomura M, Ito T, Hishida H: Use of percutaneous cardiopulmonary support of patients with fulminant myocarditis and cardiogenic shock for improving prognosis. Am J Cardiol 1999; 83: 623-625

7. Davidoff R, Palacios I, Southern J, Fallon JT, Newell J, Dec GW: Giant cell versus lymphocytic myocarditis: A comparison of their clinical features and long-term outcomes. Circulation 1991; 83: 953-961

8. Lieberman EB, Hutchins GM, Herskowitz A, Rose NR, Baughman KL: Clinicopathological description of myocarditis. J Am Coll Cardiol 1991; 18: 1617-1626

9. Cooper LT, Berry G, Shabetai R: Idiopathic giant-cell myocarditis: Natural history and treatment. N Engl J Med 1997; 336: 1860-1866

10. McCarthy RE, Boehmer JP, Hruban RH, Hutchins GM, Kasper EK, Hare JM, et al: Long-term outcome of fulminant myocarditis as compared with acute (nonfulminant) myocarditis. $N$ Engl J Med 2000; 342: 690-695

11. Aretz HT: Myocarditis: The Dallas criteria. Hum Pathol 1987; 18: 619-624

12. Japanese Circulation Society (JCS) Task Force Committee on Chronic Myocarditis: Guideline for diagnosing chronic myocarditis. Jpn Circ J 1996; 60: 263-264

13. Japanese Circulation Society (JCS) Task Force Committee on Fulminant Myocarditis: National surveillance of patients with fulminant myocarditis managed by cardiopulmonary support systems. Jpn Circ J 2000; 64(Suppl 3): 985-992 (in Japanese)

14. Mason JW, O'Connell JB, Herskowitz A, Rose NR, McManus BM, Billingham ME, et al: A clinical trial of immunosuppressive therapy for myocarditis. $N$ Engl J Med 1995; 333: 269-275

15. McNamara DM, Rosenblum WD, Janosko KM, Trost MK, Villaneuva FS, Demetris AJ, et al: Intravenous immune globulin in the therapy of myocarditis and acute cardiomyopathy. Circulation 1997; 95: 2476-2478

16. Kodama M, Okura Y, Hirono S, Hanawa H, Ogawa Y, Ito M, et al: A new scoring system to predict the efficacy of steroid therapy for patients with active myocarditis: A retrospective study. Jpn Circ J 1998; 62: 715-720 